\title{
Fortalecimiento de los procesos de lectoescritura a través de la implementación de estrategias pedagógicas apoyadas en las TIC ${ }^{1}$
}

Strengthening of the processes of lectoescritura across the
implementation of pedagogic strategies rested on the TIC

DOI: http://dx.doi.org/10.17981/cultedusoc.9.3.2018.112

Artículo de investigación. Fecha de recepción: 15/06/2018. Fecha de aceptación: 27/11/2018

\author{
Carmen Sarmiento ${ }^{2}$ \\ Liberata Ojeda ${ }^{3}$ \\ IED Juan Manuel Rudas (Colombia) \\ sharlan26@hotmail.com
}

Para citar este artículo:

Sarmiento, C. y Ojeda, L. (2018). Fortalecimiento de los procesos de lectoescritura a través de la implementación de estrategias pedagógicas apoyadas en las TIC. Cultura. Educación y Sociedad 9(3), 945-950. DOI: http://dx.doi.org/10.17981/cultedusoc.9.3.2018.1112

\section{Resumen}

El presente artículo tiene como objetivo fortalecer los procesos de lectoescritura de los estudiantes de básica primaria de la Institución Educativa Departamental Juan Manuel Rudas, a través de la implementación de estrategias pedagógicas apoyadas en TIC. Estas estrategias refieren al uso de textos literarios, trabalenguas, adivinanzas y la creación de los mismos. La metodología implementada se dio desde un enfoque cualitativo con un alcance descriptivo y un diseño fenomenológico con una población participante de 60 estudiantes. Los resultados obtenidos nos sitúan en que si se logró un avance frente a la mejora de las competencias en lectura y escritura teniendo que los estudiantes leen con más fluidez y aumento la capacidad de análisis y comprensión de textos, y por el lado de la escritura también se disminuyeron las dificultades en caligrafía y ortografía.

Palabras clave: procesos lectores, escritura, estrategias pedagógicas.

\section{Abstract}

The present article Juan Manuel Rudas has as aim strengthen the processes of reading and writing of the students of basic primary of the Educational Departmental Institution, across the implementation of pedagogic strategies rested on TIC. These strategies recount to the use of literary texts, tongue twister, riddles and the creation of the same ones. The implemented methodology there gave himself from a qualitative approach with a descriptive scope and a design phenomenon with a population participant of 60 students. The obtained results place us in that if an advance was achieved opposite to the improvement of the competitions in reading and writing having that the students read with more fluency and I increase the capacity of analysis and comprehension of texts, and for the side of the writing also the difficulties were diminished in calligraphy and spelling.

Keywords: reading processes, writing, pedagogic strategies.

\footnotetext{
1 Este artículo ha sido derivado del Programa de Fortalecimiento de la Cultura Ciudadana y Democrática CT+I a través de la IEP apoyada en TIC en el Departamento de Magdalena: CICLON
}

2 Líder del grupo de investigación "Los Ciclolectores"..

3 Docentes de la Institución Educativa Departamental Juan Manuel Rudas.

- The author; licensee Universidad de la Costa - CUC.

Cultura, Educación y Sociedad vol. 9 no. 3, pp. 945-950. Diciembre, 2018

Barranquilla. ISSN 2389-7724 Online 


\section{Introducción}

Actualmente el sistema educativo ha sufrido diversos cambios referentes a las formas en las que se puede alcanzar la transformación en el proceso de enseñanza aprendizaje (Lerner, 2001), lo anterior soporta que el hecho de implementar en la educación nuevos programas, estrategias y propuestas aporten para enfrentar las problemáticas que se vivencian a diario entre los educadores, estudiantes y comunidad educativa en general frente a las competencias lectoescritoras que se manejan. Por lo tanto, se pretende que se dé una formación a los estudiantes que los haga capaces de desarrollar las competencias ya adquiridas y que adquieran unas nuevas que le fortalezcan su proceso de aprendizaje y construcción de conocimiento (lingüística, sociolingüística, textual, discursiva, léxica entre otras) (Weiss, 2006).

En la escuela como un escenario donde se produce conocimiento se concibe a los alumnos como individuos que se deben preparar para los cambios que trae la globalización en el sistema educativo, lo que incluye la forma y modo de uso de la Lengua Castellana. Según Litwin (1997) se considera que el desarrollo de competencias en esta área contribuye a que se dé la constitución de un sujeto con habilidades críticas, reflexivas y que se perciban capaces de producir y comprender saberes de manera significativa; además le va a permitir a los niños, niñas y jóvenes prepararse para los desafíos sociales que trae consigo el medio (Rivilla, 2009).

El enfoque comunicativo aporta a la forma en cómo se concibe la Lengua Castellana hoy por hoy, teniendo en cuenta que este plantea que debe darse de manera menos formal para mejorar la comprensión e interpretación de quien lo estudia (Carlino, 2004), es decir, no se debe regir netamente a la teoría plasmada sino; que se debe concentrar la atención en el hecho de que se debe entender los que se quiere comunicar y que no es lo mismo a la enseñanza de la gramática ya que la enseñanza de la Lengua debe ser visto de manera global (Przesmycki, 1994).
Por otra parte, la Lengua castellana no puede ser impartida de manera individual vislumbrando solo el elemento gramatical ya que está constituida a partir de distintos referentes (Fuenmayor, 2007). El enfoque comunicativo centra su atención en que es y que hace el lenguaje en la interacción humana, ya que a partir de esta se determina que debilidades hay que fortalecer, contenidos se deben enseñar, actitud deben tener los aprendices, que función cumplen los maestros, que recursos, técnicas y procedimientos se utilizan (Lucio, 1989). En consecuencia, enseñar Lengua Castellana bajo un enfoque comunicativo se entiende como que los estudiantes asuman un papel de hablantes-escritores, recetoreslectores y que desde ello puedan ser reflexivos y críticos, robusteciendo procesos cognitivos como el de pensamiento, inteligencia y creatividad (Leal, 2005)

Según Serrón (2002), Franco (2007), Hymes (1964) dentro de este enfoque se manejan competencias que a su vez contienen otras competencias que se denominan subcompetencias comunicativas que le dan al estudiante habilidades para su desarrollo académico, constituyendo contenidos sociolingüísticos y contextuales que deber ser correctamente usados teniendo en cuenta aspectos como la coherencia, pertenencia y eficacia (Díaz, 2004)

Hymes (1964) relaciona que la teoría lingüística con la comunicación guarda relación en la medida que los factores socioculturales repercuten de manera directa en cómo se utiliza el lenguaje en las relaciones interpersonales, entonces parte de allí que el estudiante pueda anclar con sus competencias en la comunidad educativa y a sus exigencias (Serrón, 2002).

Todos los conceptos manejados denotan que las competencias comunicativas referente al uso adecuado de la Lengua Castellana se enmarcan en el contexto donde se esté dando la comunicación y cuales sean las normas que estén establecidas y cuáles son las estrategias que se exigen para que se dé (Franco, 2007). En este sentido García y Arrieta (2016), destacan la necesidad de integrar estrategias 
didácticas cognitivas y metacognitivas como base para desarrollar el pensamiento crítico a través del lenguaje. Estas dependen de la diversa y compleja interacción de competencias o sub-competencias estas corresponden a distintos autores a las siguientes; Competencia lingüistica, Competencia discursiva, Competencia situacional, Competencia semiolingüistica, Competencia sociocultural (sociolingüistica) y Competencia pragmática.

\section{Lectoescritura}

La lectoescritura es un medio fundamental para que los estudiantes mejoren sus competencias de lenguaje, esta se imparte desde los años de preescolar, como educación inicial, en la básica primaria y en la secundaria impulsando a que se identifique cual es la relación entre los procesos de lectura y escritura (Ardila, 2016); sin embargo se alude que las dificultades en este proceso pueden presentarse durante la primara ya que los contenidos se complejizan por lo que se requiere que las bases de formación sean las adecuadas y puedan enfrentar los desafíos que trae consigo el aprendizaje del lenguaje escrito y oral (Camilloni, 1998). Autores como Pinto y Misas (2014) destacan que los primeros niveles del sistema educativo constituyen la base para el desarrollo de competencias que orienten procesos de formación integral centrados en la autonomía y la estimulación del estudiante.

La adquisición y la forma en cómo se han constituido las bases conceptuales de los estudiantes van a determinar su desenvolvimiento, tanto en el medio académico como con los aspectos culturales; además se suman elementos como el desarrollo de los procesos psicológicos como la percepción, la memoria, la cognición, la metacognición, capacidad inferencial, y pensamiento (Montealegre, 2006). Aunado a esta postura destacan los aportes de Pino y Urrego (2013) y Moreno (2015) cuando conciben que el desarrollo del individuo está imbricado por el contexto, es decir, los procesos formativos deben apuntar a la integralidad entre los diferentes componentes académicos, sociales y personales.

Si nos adentramos a la situación que vive la Institución Educativa Departamental Juan
Manuel Rudas en cuanto al proceso de lectoescritura que realizan sus estudiantes, solo podemos ver escenas de apatía, cansancio en inclusive rechazo particularmente entre los niños de tercer y cuarto de primaria, situación que se refleja en su bajo rendimiento académico particularmente en la asignatura de lenguaje, mostrando grandes deficiencias en cuanto a la lectura y la escritura. Por lo tanto, la forma de enseñanza de los procesos lectoescritores debe hacerse en escenarios propicios con herramientas y actividades lúdicas que generen motivaciones en los alumnos. (Mora, 2005).

Dentro de la población estudiantil los maestros investigadores han identificado que las debilidades más relevantes son:

- Deficiencias al separar palabras o silabas

- No saber cómo separar las frases

- Omitir letras al escribir frases

- Los textos carecen de sentido con fallas gramaticales y ortográficas

- Confusión en algunas consonantes

- Al leer texto lo hacen de manera silábica, no frases completas.

- Falta de comprensión lectora en textos cortos y sencillos.

Todas estas situaciones mencionadas anteriormente y el interés de los docentes por superarlas, llevaron a la creación e implementación de este proyecto que tuvo la finalidad de contrarrestar el alto índice de bajo rendimiento académico en la lectoescritura, de manera, que se pueda conocer e identificar las dificultades y emplear estrategias acordes para dar sus posibles soluciones.

\section{Metodología}

La investigación realizada fue desarrollada desde un enfoque cualitativo con un alcance descriptivo con un diseño fenomenológico teniendo en cuenta que se pretende identificar y describir una problemática e intervenir en ella (Hernández Sampieri, 2014), en este caso los maestros tienen como objetivo facilitar a los estudiantes el aprendizaje de la lectoescritura a través de la implementación 
de estrategias lúdico-pedagógicas que le permitan fortalecer al final sus competencias en expresión oral y escrita.

\section{Participantes}

Durante el estudio participaron 60 estudiantes de la Institución Educativa Departamental Juan Manuel Rudas, distribuidos en los cursos pertenecientes a la básica primaria, con edades que oscilaron entre los 4 y 11 años.

\section{Técnicas e instrumentos de recolección de información}

Las técnicas de recolección de información elegidas para la aplicación del estudio fue la observación participante debido a que los maestros además de registrar los comportamientos, actitudes y avances de los estudiantes participando en la aplicación de las actividades pedagógicas diseñadas ya que el investigador puede inmiscuirse para escuchar y observar el fenómeno en tiempo y espacio real (Hernández Sampieri, 2014). Como instrumento se selecciona el diario de campo donde se registrarán todos los aspectos importantes de la intervención.

\section{Procedimiento}

Las actividades diseñadas por los investigadores están contempladas en la realización de talleres en los cuales se llevarán a cabo actividades enfocadas al fortalecimiento de las competencias lectoescritoras. Los talleres se realizaron una vez por semana en horas de la tarde como jornada complementaria con los estudiantes participantes en ellos se requirieron recursos como cartulinas, marcadores, lápices, colores, entre otros. Además, en los talleres los maestros se valían también de herramientas como cuentos, videos de Youtube y aparatos tecnológicos para apoyarse en las tecnologías de la información y comunicación. Posterior a ello se socializarán los resultados y experiencias de los estudiantes por medio de cartillas, grabaciones y un periódico mural.

\section{Resultados}

En el siguiente apartado se exponen los resultados obtenidos a partir de la implementación de los talleres planteados para la aplicación del estudio (Tabla 1).

TABLA 1

Resultados obtenidos

\begin{tabular}{|c|c|c|}
\hline Sesión & Actividad & Resultado \\
\hline $\begin{array}{l}\text { Sesión } \\
1\end{array}$ & $\begin{array}{l}\text { Taller "aprende } \\
\text { la lectura" }\end{array}$ & $\begin{array}{l}\text { En la presente actividad a los estudiantes se les organizo por grupos y se les asigno a } \\
\text { cada uno una lectura que debían aprender sobre adivinanzas y trabalenguas, con ellos se } \\
\text { realizó un concurso donde el ganador era el grupo que dijera el trabalenguas de manera } \\
\text { correcta y quien acertara la adivinanza, se le proporcionaron los textos impresos para } \\
\text { la respectiva lectura. A los estudiantes durante la actividad se les noto motivados y } \\
\text { entusiastas, y manifestaban que les gustaba la estrategia porque era una clase diferente } \\
\text { y podían divertirse con los compañeros, en este mismo orden los maestros identificaron } \\
\text { que los niños avanzaron en la habilidad de memorizar textos medianamente extensos y el } \\
\text { ritmo de lectura. }\end{array}$ \\
\hline Sesión 2 & $\begin{array}{l}\text { Taller "Historias, } \\
\text { palabras e } \\
\text { imágenes" }\end{array}$ & $\begin{array}{l}\text { En la presente actividad a los estudiantes se les indicó que a partir de la lectura del } \\
\text { texto proporcionado debían inicialmente determinar cuál era la situación que se vivía } \\
\text { en la historia y que lugares se encontraban en ella y al final dibujar el lugar que más les } \\
\text { haya gustado. Durante la actividad los estudiantes manifestaron gustarle el hecho de } \\
\text { tener que buscar datos en el texto y como actividad final dibujar, ya que podían colorear } \\
\text { y pintar, asimismo los maestros observaron que en los grupos había alto interés por la } \\
\text { actividad y la lectura. }\end{array}$ \\
\hline
\end{tabular}




\begin{tabular}{|c|c|c|}
\hline Sesión 3 & $\begin{array}{l}\text { Taller "búsqueda } \\
\text { del tesoro de } \\
\text { letras y formar } \\
\text { palabras" }\end{array}$ & $\begin{array}{l}\text { En este taller a los estudiantes se les explico que se realizaría una carrera de obstáculos } \\
\text { en la que encontrarían a su paso unos cartones con letras dibujadas al final de la pista, } \\
\text { ellos debían adivinar que palabra se formaba con las letras y armar al menos } 3 \text { palabras } \\
\text { más al grupo ganador se le obsequiaba un dulce como premio. En este orden los maestros } \\
\text { identificaron la motivación de los estudiantes y que a través de este tipo de actividades se } \\
\text { canaliza su atención y aumenta la participación, además expresaban que la lectura para } \\
\text { ellos si era trabajada de esta manera era más divertida y sentían que aprendían más. }\end{array}$ \\
\hline Sesión 4 & $\begin{array}{l}\text { Taller "YouTube } \\
\text { para estudiar" }\end{array}$ & $\begin{array}{l}\text { Por medio de la plataforma audiovisual YouTube y haciendo uso de las tabletas de la } \\
\text { institución los estudiantes observaron dos videos acerca del cuento de "caperucita roja" de } \\
\text { este debían realizar un análisis crítico en el que identificaron aspectos como, recorrido del } \\
\text { personaje, objetivo del lobo y enseñanza de la historia. En esta actividad los estudiantes } \\
\text { se sintieron entretenidos y concentrados teniendo en cuenta que el recurso didáctico } \\
\text { utilizado fue la tablet por niño generando así empoderamiento en su desarrollo. }\end{array}$ \\
\hline Sesión 5 & $\begin{array}{l}\text { Taller "mi } \\
\text { amigo por } \\
\text { correspondencia" }\end{array}$ & $\begin{array}{l}\text { La dinámica establecida para el taller fue organizar por grupos a los participantes y } \\
\text { cada uno se les denomino con el nombre de un país del mundo, entonces se les entrego } \\
\text { hojas de block y lápiz para que le redactaran una carta a un amigo que se encontraba en } \\
\text { otro país, y al final esta carta debía ser leída e intercambiada entre grupos (remitente y } \\
\text { receptor). Posteriormente los maestros leyeron los escritos de los niños y determinaron } \\
\text { las dificultades de ortografía y caligrafía, pero se evidencio una mejora significativa en la } \\
\text { lectura observando que hay más fluidez y comprensión de textos a nivel general. }\end{array}$ \\
\hline
\end{tabular}

Fuente: elaboración propia.

Finalmente la socialización de los resultados alcanzados en los talleres se socializo con la comunidad estudiantil en general a través de una cartilla donde los estudiantes por grupos debían escribir como se sintieron en las actividades y los avances de sus competencias lectoescritoras, en esta parte los estudiantes plantearon que se sintieron a gusto en cada actividad ya que, para ellos fue divertido aprender por medio de concursos y estrategias diferentes a las clases tradicionales. Por otra parte en las publicaciones del periódico mural se informó a la demás comunidad educativa los hallazgos y datos relevantes de la investigación, entre los que resaltan la motivación de los estudiantes frente a las actividades, los progresos en el área de Lengua Castellana y por ende mejora en el rendimiento académico, además se proyectó a los docentes y directivos las evidencias grabadas en los talleres por los maestros investigadores explicando el objetivo y el éxito del uso de estrategias pedagógicas basadas en TIC.

\section{Discusión}

El desarrollo de temáticas de impacto en la institución desde el uso de estrategias lúdico- pedagógicas llegan a ser las herramientas fundamentales para despertar en los niños, $\mathrm{y}$ jóvenes el interés en este caso por la lectura y la escritura aportando sus ideas y esfuerzo al proceso de mejora. En este sentido se agrega que de manera implícita se fomenta el trabajo investigativo, se aumenta la capacidad de análisis y la autoevaluación en el aprendizaje originando en los estudiantes un rol activo.

El proyecto ejecutado concluyó como un medio por el cual se genera más participación de los estudiantes teniendo en cuenta que aumenta la producción de nuevos saberes y se fortalecen conocimientos previos y también se extraen de sus experiencias lo más beneficioso apuntando a que este siempre sea constructor y el maestro un guía.

Por lo anterior los investigadores sugieren que los padres como actores también de la comunidad estudiantil, hagan parte de las actividades, con el fin de que hagan el trabajo independiente en casa y en conjunto con los estudiantes solidificar las debilidades, entonces así una de las estrategias podría ser el uso de cuentos típicos de la región por ejemplo, mitos y leyendas; lo cual contribuye a clarificar las temáticas dadas en el plan curricular y se aprovecha también el saber popular de los padres como acompañantes. 


\section{Referencias}

Acevedo, C. (2017). Las tiendas de barrio desde la economía institucional. IJMSOR, 2(1), 30-37. Recuperado de http://ijmsoridi.com/index.php/ijmsor/article/ view/85

Addine, F., González, A. y Recarey, S. (2002). Principios para la dirección del proceso pedagógico. Compendio de pedagogía, 80-101.

Ardila, J. (2016). Lectura y escritura. Paideia Surcolombiana, (6). 71-74.

Arnáez, P. (2001). El componente lingüístico en un modelo comunicacional. Letras, (63). 123-144.

Carlino, P. (2004). El proceso de escritura académica: cuatro dificultades de la enseñanza universitaria. Educere, 8(26). 321-327.

Camilloni, A., Davini, M., Edelstein, G., Litwin, E., Souto, M. y Barco, S. (1998). Corrientes didácticas contemporáneas. Buenos Aires: Paidós.

Díaz, T. (2004). Temas sobre pedagogía y didáctica de la Educación Superior. Medellín: Fundación Educativa Esumer.

Fuenmayor, G. (2007). Metodología lingüística para el logro de los niveles de comprensión lectora de textos expositivos de estudiantes universitarios. [Tesis doctoral]. Universidad Rafael Belloso Chacín, Maracaibo, Venezuela.

Franco, A. (2007). Gramática Comunicativa. Maracaibo: Vicerrectoría Académica.

García, H. y Arrieta, A. (2016). Potencialización del Pensamiento Crítico a través de la Hipotemetacomprensión Textual (Hmt). Cultura Educación y Sociedad, 7(2), 54-71. Recuperado vde https://revistascientificas.cuc.edu.co/culturaeducacionysociedad/article/ view/1102

García, J. y Nando, J. (2000). Estrategias didácticas en educación ambiental. Edetania 40(1). 15-31.

Hernández, D. y Escobar, A. (2017). Modelo de contabilidad social como herramienta de gestión para la responsabilidad social empresarial. IJMSOR, 2(1), 44-56. Recuperado de http://ijmsoridi.com/index. php/ijmsor/article/view/86

Hymes, D. (1964). Hacía la etnografía de la comunicación. En, P. Garvín y Y. Lastra (Comps). Antología de estudios de etnolingüistica y sociolingüística. (4890). México, D.F.: UNAM.

Leal, A. (2005). Didáctica, pedagogía y saber. Bogotá, D.C.: Magisterio.

Lerner, D. (2001). Leer y escribir en la escuela: lo real, lo posible y lo necesario. México, D.F.: FCE.

Litwin, E. (1997). Las Configuraciones Didácticas. Buenos Aires: Paidós.

Lucio, R. (1989). Educación y pedagogía, enseñanza y didáctica: diferencias y relaciones. Revista Universidad de la Salle, (17), 35-46.
Mora, D. (2005). Didáctica crítica, educación crítica de las matemáticas y etnomatemática. La Paz: Campo Iris.

Moreira, M. (2004). Los medios y las tecnologías en la educación. Madrid: Pirámide.

Ochoa, R. (1994). Hacia una pedagogía del conocimiento. Bogotá, D.C.: McGraw-Hill.

Przesmycki, H. (1994). La Pedagogía de contrato: el contrato didáctico en la educación. Barcelona: Graó.

Montealegre, R. y Forero, L. (2006). Desarrollo de la lectoescritura: adquisición y dominio. Acta Colombiana de Psicología, 9(1). 25-40.

Moreno, G. (2015). Una Aproximación al concepto de Observatorio Social. Cultura Educación y Sociedad, 6(1). 93-108. Recuperado de https://revistascientificas.cuc.edu.co/culturaeducacionysociedad/article/ view/758

Pino, M. y Urrego, Y. (2013). La importancia de las funciones ejecutivas para el desarrollo de las competencias ciudadanas en el contexto educativo. Cultura Educación y Sociedad, 4(1). 9-20- Recuperado de https://revistascientificas.cuc.edu.co/culturaeducacionysociedad/article/view/969

Pinto, M. y Misas, M. (2014). La educación inicial y la educación preescolar: perspectivas de desarrollo en Colombia y su importancia en la configuración del mundo de los niños. Cultura Educación y Sociedad, 5(2). 119-140. Recuperado de https://revistascientificas.cuc.edu.co/culturaeducacionysociedad/article/ view/889

Rivilla, A. y Mata, F. (Coord.) (2009). Didáctica general. Madrid: Pearson.

Sagastume, A. y Cabello, J. (2017). La educación superior y una producción más limpia. IJMSOR, 2(1), 4-8. Recuperado de http://ijmsoridi.com/index.php/ijmsor/ article/view/79

Segura, E. (2016). Información, estabilidad y complejidad de aprendizaje en memorias asociativas. IJMSOR, 1(1), 49-53. Recuperado de http://ijmsoridi. com/index.php/ijmsor/article/view/77

Sepúlveda, A., Martínez, R., Medina, S. y Salazar, F. (2016). Propuesta de diseño de una red supply chain para la agrocadena de cacao, municipio de Viotá, Cundinamarca. IJMSOR, 1(1), 35-42. Recuperado de http://ijmsoridi.com/index.php/ijmsor/article/ view/75

Serrón, S. (2001). (2do semestre). El enfoque comunicativo y sus implicaciones. Una visión desde la enseñanza de la lengua materna. Letras. (63). 83-84.

Valencia, F. (2017). Lectura, incertidumbre, escritura. Revista Cintex, 3, 15-21.

Vasco, C. (1990). Algunas reflexiones sobre la pedagogía y la didáctica. Pedagogía, discurso y poder. 107-122.

Weiss, E. (2006). Los jóvenes como estudiantes. Revista mexicana de investigación educativa, 11(29). 359. 366. 